\title{
Fusarium head blight (FHB) and Fusarium spp. on grain of spring wheat cultivars grown in Poland
}

\author{
Leszek Lenc ${ }^{*}$, Grzegorz Czecholinski², Dariusz Wyczling ${ }^{3}$,Tomasz Turów ${ }^{3}$, \\ Arkadiusz Kaźmierczak ${ }^{1}$
}

\author{
${ }^{1}$ Department of Molecular Phytopathology, University of Technology and Life Sciences in Bydgoszcz, \\ Kordeckiego 20, 85-225 Bydgoszcz, Poland \\ ${ }^{2}$ Experimental Station for Variety Testing in Lisewo Malborskie, Kolejowa 43, 82-224 Lichnowy, Poland \\ ${ }^{3}$ ProCam Polska Sp. z o.o., Nowy Świat 42/44, 80-299 Gdańsk, Poland
}

Received: April 9, 2015

Accepted: June 17, 2015

\begin{abstract}
Eighteen spring wheat cultivars, recommended for commercial production in northern Poland, were assessed for Fusarium head blight (FHB) in natural non-epidemic conditions, from 2011 to 2013. Assessment was based on FHB incidence (proportion of heads with symptoms), disease severity (DS; proportion of bleached spikelets per head), proportion of Fusarium damaged kernels (FDK), and spectrum of Fusarium spp. colonising the kernels. Fusarium head blight incidence and DS often differed significantly among cultivars and years. There was a strong positive correlation between FHB incidence and DS. Fusarium head blight incidence and DS were not correlated with the June-July temperatures, and were only occasionally correlated with the total June-July rainfall. There was a weak positive correlation between FHB incidence and proportion of FDK. There was a strong positive correlation between DS and proportion of FDK. The cultivar affected colonisation of kernels by Fusarium spp. Fusarium poae was the FHB pathogen isolated most often. Fusarium poae colonised $6.0 \%$ of the kernels, on average, but up to $12.0 \%$ on individual cultivars. Other Fusarium species were less frequent: F. avenaceum in $5.6 \%$ of kernels, F. culmorum in $5.3 \%$, F. tricinctum in $2.8 \%$, F. graminearum in $1.5 \%$, and F. sporotrichioides in $1.2 \%$. Fusarium equiseti occurred sporadically. The importance of $F$. poae in the FHB complex is emphasised. All cultivars expressed 'moderate FHB resistance' if evaluated according to FHB incidence. Cultivars Arabella, Izera, Kandela, Monsun, Ostka Smolicka, and Struna expressed 'moderate susceptibility', and Bombona, Hewilla, Katoda, KWS Torridon, Łagwa, Nawra, Parabola, Radocha, SMH 87, Trappe, Tybalt, and Waluta expressed 'susceptibility' if evaluated by the proportion of FDK. Cultivars differed within the 'moderately resistant', 'moderately susceptible', and 'susceptible' categories. Cultivars Arabella, Izera, Kandela, Monsun, Ostka Smolicka, and Struna were the most promising and their resistance traits may be useful in FHB management.
\end{abstract}

Key words: cultivar, Fusarium, Fusarium head blight (FHB), spring wheat, susceptibility

\section{Introduction}

Spring wheat is the second most valuable cereal after winter wheat. It is grown in Poland on 380000 ha, particularly in the north and in Lower Silesia (southern Poland). The area has increased recently because of losses occurring in winter crops. Spring wheat's advantages make it popular in large-scale and commercial production, and locally on ecological farms.

Spring wheat cultivars bred in Europe are characterised by short, lodging-resistant stalks (usually decreasing FHB, which can be more severe in crops that lodge), higher yields, and high efficiency in mineral nutrition. The quality of the grain depends on the genetic features of the cultivar, weather conditions, and agricultural management. Spring wheat may have less yield than its winter equivalent. Instead, the popularity of spring wheat results from its agricultural value. Spring wheat has a shorter period of growth and of exposure to pathogens, pests, and weeds. All of these features decrease cultivation costs.

Fusarium head blight (FHB) is one of the most important cereal diseases. It has emerged as a major threat to wheat and barley crops around the world. Fusarium head blight contributes to loss of grain yield and quality due to colonisation by Fusarium fungi and contamination with mycotoxins (Siuda et al. 2010; Grabowski et al. 2012a, b; Gromadzka et al. 2012).

The prevalence of disease depends on agronomic practices, effectiveness of fungicides used, and host resistance. In Poland, FHB has been observed every year with differences in incidence. In some years, incidence was low ( $<1 \%$ of heads colonized). Recently, FHB severity has increased, and has been seen each year on approximately $70 \%$ of wheat fields (Wakuliński et al. 1991; Lenc et al. 2011; Sadowski et al. 2011; Lenc 2015). 
Seventeen species of Fusarium, with different climatic requirements and genetic and environmental adaptations, contribute to disease (Parry et al. 1995; Stępień and Chełkowski 2010; Wiśniewska et al. 2014). Fusarium graminearum Schwabe [Gibberella zeae (Schwein.) Petch] is the predominant causal agent of FHB in most areas. This fungus is the most virulent worldwide. In Poland, FHB is caused mostly by F. culmorum (Wm. G. Sm.) Sacc., F. avenaceum (Fr.) Sacc. (Gibberella avenacea R.J. Cook) and F. poae (Peck) Wollenw. (Wakuliński and Chełkowski 1993; Lenc 2015). The contribution of particular species to FHB incidence depends on habitat and weather conditions.

Deoxynivalenol (DON) and zearalenone are the most common mycotoxins associated with FHB They pose a serious threat to human and domestic animal health. Crop sequence and tillage (which incorporates crop residues into the soil) have been shown to affect the incidence of FHB. In recent years, decreased tillage is thought to have contributed to regional epidemics by increasing the levels of inoculum available for infection. Additionally, disease is favored by extended periods of moderately high temperature $\left(15-30^{\circ} \mathrm{C}\right)$, high moisture (relative humidity $>90 \%$ ), and frequent rainfall (De Wolf et al. 2003; Lemmens et al. 2004; Xu et al. 2008; Sadowski et al. 2011; Lenc 2015).

Extensive research aimed at controlling FHB has focused on the development of wheat cultivars resistant to Fusarium spp. and the use of such resistant cultivars in integrated management systems. The development of resistant cultivars is the most effective, economic, and environmentally safe way to control FHB in wheat. Thousands of plant lines are subjected to artificial inoculation with Fusarium spp. (mostly with F. graminearum). Those lines having reduced fungal growth and low levels of seed contamination with DON, are selected and advanced in further breeding trials. Quantitative Trait Loci (QTL) composed of one or more genes, such as Fhb1 derived from the Chinese wheat cultivar Sumai 3, have been identified and widely used in wheat breeding (Steiner et al. 2004; Góral 2005; Zhang et al. 2008; Kubo et al. 2013).

Two main types of resistance to FHB are widely accepted: Type I, resistance to initial infection; and Type II, resistance to fungal spread within the spike (Schroeder and Christensen 1963). Three other types of resistance were reported by Mesterhazy et al. (1999): Type III, resistance to DON accumulation; Type IV, resistance to kernel infection; Type $\mathrm{V}$, tolerance. Infection and disease development can be affected by both active (i.e. physiological processes) and passive (i.e. plant height, spike architecture, flowering date) resistance factors that are difficult to separate (Crute et al. 1985; Mesterhazy 1995; Buerstmayr et al. 2009). Integrated management of FHB in wheat depends on disease forecasting models which help to determine the risk of FHB infection and optimise the agronomic and chemical control of disease. The risk of FHB infection depends partly on cultivar resistance to Fusarium spp., which must be considered in commercial production. Genetic variability is essential for the development of FHB resistant cultivars. Sources of FHB resistance/tolerance in spring wheat have so far been introduced from China, Brazil, Europe, and Japan (Fedak et al.
2001). There is always a chance of finding further sources of resistance in local cultivars and lines.

The objective of this study was to assess: (i) FHB incidence and disease severity in 18 cultivars of spring wheat grown in a conventional system in northern Poland in the 2011-2013 time period; (ii) effects of weather conditions on FHB incidence and severity; (iii) populations of $\mathrm{Fu}$ sarium fungi involved in FHB, and hence, implications for human and domestic animal mycotoxicoses; (iv) suitability of particular spring wheat cultivars for FHB resistance breeding, commercial production, and requirements of integrated management. The objectives are of particular significance in terms of the European Commission Regulation No. 856/2005 of 6 June 2005 regarding food safety and Fusarium toxin concentrations in food and feed.

\section{Materials and Methods}

\section{Site description}

Seventeen common spring wheat (Triticum aestivum L.) cultivars (Arabella, Bombona, Hewilla, Izera, Kandela, Katoda, KWS Torridon, Łagwa, Monsun, Nawra, Ostka Smolicka, Parabola, Radocha, Struna, Trappe, Tybalt, and Waluta) from elite groups (A-E), and one durum spring wheat (T. turgidum ssp. durum) cultivar (SMH 87) were grown in a conventional system in experimental fields at the Experimental Station for Variety Testing in Lisewo Malborskie, northern Poland (54 $6^{\prime} \mathrm{N}, 18^{\circ} 43^{\prime} \mathrm{E}$ ) from 2011 to 2013. Not all cultivars were grown in each of the study years. The experiment was established with four replicates in a randomised block design in brown soil of class I quality. The chemical characteristics of the soil at Lisewo (2011-2013) are presented in table 1. The previous crop in each year was the sugar beet (Beta vulgaris L.). Sowing was done on 8-10 April at 450-500 grains $\cdot \mathrm{m}^{-2}$. Fertiliser application each year was: $140 \mathrm{~kg} \mathrm{NH}_{4}^{+} \cdot \mathrm{ha}^{-1}$, $70 \mathrm{~kg} \mathrm{P}_{2} \mathrm{O}_{5} \cdot \mathrm{ha}^{-1}$ and $100 \mathrm{~kg} \mathrm{~K}_{2} \mathrm{O} \cdot \mathrm{ha}^{-1}$. Fungicidal seed treatments, and herbicide and insecticide sprays were applied each year (Table 2). Grain was harvested each year on 27-30 August.

The average 2011-2013 temperatures in June were $15.5-17.8^{\circ} \mathrm{C}$, and in July $18.3-18.6^{\circ} \mathrm{C}$, with the highest temperatures in July 2012 (Table 3). Total rainfall in June was from 36.2 to $125.1 \mathrm{~mm}$, and in July from 102.3 to $170.3 \mathrm{~mm}$, with the most rain in July 2013. The number of days with rain was in the range of 8-18 in June, and 12-20 in July. Although temperatures were moderate in JuneJuly 2011-2013, the high rainfall in June 2012 and July 2011-2013, and the high number of days with rainfall, particularly in July 2011-2013, meant that weather conditions were generally favorable for FHB development.

\section{Collection of samples and disease assessment}

Each year (2011-2013), 50 heads of wheat from each cultivar were collected at late milk to early dough development stages (GS 77-83; Zadoks et al. 1974). These wheat heads were collected from randomly chosen plants along a diagonal transect across each of the four replicate plots. Fusarium head blight incidence in heads, and disease se- 
Table 1. Chemical characteristics of soil at Lisewo, in 2011-2013

\begin{tabular}{lccc}
\hline \multicolumn{1}{c}{ Soil characteristics } & 2011 & 2012 & 2013 \\
\hline $\mathrm{pH}$ in $\mathrm{KCl}$ & 5.9 & 5.8 & 6.2 \\
Extractable soil phosphorus $\left[\mathrm{mg} \cdot \mathrm{kg}^{-1}\right]^{\mathrm{a}}$ & 9.9 & 24.4 & 18.2 \\
Extractable soil potassium $\left[\mathrm{mg} \cdot \mathrm{kg}^{-1}\right]^{\mathrm{b}}$ & 17.8 & 20.0 & 25.1 \\
Extractable soil magnesium $\left[\mathrm{mg} \cdot \mathrm{kg}^{-1}\right]^{\mathrm{b}}$ & 12.6 & 12.4 & 13.5 \\
\hline
\end{tabular}

aanalysed with the Egner-Riehm method

banalysed with the Schachtschabel method

Table 2. Pesticides used in spring wheat production at Lisewo, in 2011-2013

\begin{tabular}{|c|c|c|c|}
\hline Seed treatment & 2011 & 2012 & 2013 \\
\hline Fungicide & $\begin{array}{l}\text { Sarfun T } 65 \mathrm{DS}^{1} \\
\left(200 \mathrm{~g} \cdot 100 \mathrm{~kg}^{-1}\right)\end{array}$ & $\begin{array}{l}\text { Sarfun T } 65 \mathrm{DS} \\
\left(200 \mathrm{~g} \cdot 100 \mathrm{~kg}^{-1}\right)\end{array}$ & $\begin{array}{c}\text { Zaprawa T 75DS/WS2 } \\
\left(200 \mathrm{~g} \cdot 100 \mathrm{~kg}^{-1}\right)\end{array}$ \\
\hline Herbicide & $\begin{array}{c}\text { Gold } 450 \mathrm{EC}^{3}+\text { Gallaper } 200 \mathrm{EC}^{4} \\
\left(1.01+0.81 \mathrm{ha}^{-1}\right)\end{array}$ & $\begin{array}{l}\text { Granstar Ultra }{ }^{5}+\text { Tomigan } 250 \mathrm{EC}^{6} \\
\quad\left(40 \mathrm{~g}+0.51 \mathrm{ha}^{-1}\right)\end{array}$ & $\begin{array}{l}\text { Granstar Ultra + Hurler } 200 \mathrm{EC}^{7} \\
\qquad\left(40 \mathrm{~g}+0.41 \mathrm{ha}^{-1}\right)\end{array}$ \\
\hline Insecticide & $\begin{array}{l}\text { Karate Zeon 050CS } \\
\quad\left(0.12 \mathrm{l} \cdot \mathrm{ha}^{-1}\right)\end{array}$ & $\begin{array}{l}\text { Karate Zeon 050CS } \\
\quad\left(0.11 \cdot \mathrm{ha}^{-1}\right)\end{array}$ & $\begin{array}{l}\text { Wojownik 050SC } \\
\left(0.11 \cdot \mathrm{ha}^{-1}\right)\end{array}$ \\
\hline
\end{tabular}

${ }^{1}$ Carbendazim 20\% + thiuram 45\%; ${ }^{2}$ Thiuram 75\%; ${ }^{3}$ Terbuthrine + metolachlor; ${ }^{4}$ Fluoroxypyr; ${ }^{5}$ Tribenuron methyl $50 \%+$ trisodium phosphate dodecahydrate 20\%; ${ }^{6}$ Fluroxypyr $25 \%$; ${ }^{7}$ Fluroxypyr meptyl 20\%; ${ }^{8}$ Lambda-cyhalothrin + 1,2-benzisothiazolin-3-one; ${ }^{9}$ Lambda cyhalothrin $5 \%$

Table 3. Temperature and rainfall during the flowering and ripening stages of spring wheat growth at Lisewo, in 2011-2013 (according to Experimental Station for Variety Testing in Lisewo Malborskie)

\begin{tabular}{|c|c|c|c|c|c|c|c|c|c|c|}
\hline \multirow{2}{*}{ Month } & \multirow{2}{*}{ 10-day period } & \multicolumn{3}{|c|}{ Average temperature $\left[{ }^{\circ} \mathrm{C}\right]$} & \multicolumn{3}{|c|}{$\begin{array}{c}\text { Total rainfall } \\
{[\mathrm{mm}]}\end{array}$} & \multicolumn{3}{|c|}{ Number of days with rainfal } \\
\hline & & 2011 & 2012 & 2013 & 2011 & 2012 & 2013 & 2011 & 2012 & 2013 \\
\hline \multirow{4}{*}{ June } & I & 19.1 & 13.0 & 15.8 & 9.1 & 16.7 & 5.6 & 3 & 6 & 2 \\
\hline & II & 16.5 & 16.9 & 18.7 & 18.1 & 24.9 & 0.5 & 4 & 6 & 2 \\
\hline & III & 17.7 & 16.8 & 17.8 & 11.7 & 83.5 & 30.1 & 2 & 6 & 4 \\
\hline & average & 17.8 & 15.5 & 17.4 & 38.9 & 125.1 & 36.2 & 9 & 18 & 8 \\
\hline \multirow{4}{*}{ July } & I & 17.6 & 20.0 & 18.2 & 47.0 & 60.6 & 88.8 & 6 & 8 & 4 \\
\hline & II & 19.4 & 16.2 & 17.4 & 45.9 & 23.8 & 38.2 & 6 & 8 & 4 \\
\hline & III & 18.2 & 19.6 & 19.4 & 15.2 & 17.9 & 43.3 & 6 & 4 & 4 \\
\hline & average & 18.4 & 18.6 & 18.3 & 108.1 & 102.3 & 170.3 & 18 & 20 & 12 \\
\hline
\end{tabular}

verity, were evaluated visually on 200 heads from each cultivar. Fusarium head blight incidence was determined as the proportion (\%) of heads with symptoms. Disease severity (DS), i.e. the extent of head damage (\%), was determined as the proportion of bleached spikelets per head, based on a 1-9 scale: $1-$ no symptoms; $2-<5 \%$ of bleached spikelets; $3-5-15 \% ; 4-16-25 \%$; 5 - 26-45\%; 6 46-65\%; 7 - 66-85\%; 8 - 86-95\%; 9 - 96-100\% (Miedaner and Perkowski 1996).

\section{Colonisation of wheat kernels by fungi}

Mycological analysis of $400(4 \times 100)$ wheat kernels collected randomly during harvest from the four plots of each cultivar was performed each of the study years. In the laboratory, the kernels were rinsed for $45 \mathrm{~min}$ in run- ning water, disinfected in $1 \% \mathrm{NaOCl}$ solution for $2.5 \mathrm{~min}$, rinsed three times for $10 \mathrm{~min}$ in sterile distilled water, and placed on Potato Dextrose Agar (PDA; boiled and sieved white potatoes $400 \mathrm{~g} \cdot \mathrm{l}^{-1}$, agar $20 \mathrm{~g} \cdot \mathrm{l}^{-1}$, streptomycin $50 \mathrm{mg} \cdot \mathrm{1}^{-1}, \mathrm{pH}=7$ ) in Petri dishes. Fungi were incubated for $7-10$ days at $20^{\circ} \mathrm{C}$ in a day-night cycle. All colonies on each plate were then examined macro- and microscopically and distinguished on the basis of colour, growth rate, hyphal characteristics, and sporulation. Colonies of each species were counted and representative fungi were identified by morphotyping on PDA and Synthetic Nutrient Agar (SNA; $\mathrm{KH}_{2} \mathrm{PO}_{4} 1 \mathrm{~g} \cdot \mathrm{l}^{-1}, \mathrm{KNO}_{3} 1 \mathrm{~g} \cdot \mathrm{l}^{-1}, \mathrm{MgSO}_{4}$. - $7 \mathrm{H}_{2} \mathrm{O} 0.5 \mathrm{~g} \cdot \mathrm{1}^{-1}, \mathrm{KCl} 0.5 \mathrm{~g} \cdot \mathrm{l}^{-1}$, glucose $0.2 \mathrm{~g} \cdot \mathrm{l}^{-1}$, sucrose $0.2 \mathrm{~g} \cdot \mathrm{1}^{-1}$ ) using Booth (1971), Kwaśna et al. (1991). Proportions (\%) of Fusarium-colonised kernels (FDK) were calculated. 


\section{Evaluation of cultivar response to Fusarium infection}

The response of each cultivar was determined from FHB incidence and the proportion of FDK. For response based on the FHB incidences (proportion of heads with symptoms), the following scale was used: 0 - immune; 1-5\% - resistant; 5-25\% - moderately resistant; $25-50 \%$ - moderately susceptible; $50-75 \%$ - susceptible; $>75 \%$ very susceptible. For response based on the proportion of FDK, the scale was as follows: 0 - immune; $1-8 \%-$ resistant; $9-11 \%$ - moderately resistant; $12-20 \%$ - moderately susceptible; $21-50 \%$ - susceptible; > 50\% - very susceptible.

\section{Statistical analysis}

The statistical significance of differences in FHB incidence and in DS on different cultivars was tested using one-way analysis of variance (ANOVA, $p \leq 0.05$ ) and Tukey's post hoc test, (FR-ANALWAR software). Percentage values were transformed into Bliss degrees before statistical analysis. The statistical significance of differences in the number of FDK and the statistical significance of differences in the number of kernels colonised by individual Fusarium species were determined using $\chi^{2}$ tests. The null hypothesis assumed that wheat from different systems had the same number of kernels colonised by Fusarium spp. Pearson's correlation coefficient was applied to analyse the relationships between FHB incidence, DS, proportion of FDK, yield, temperature, and rainfall.

\section{Results}

\section{Effects of cultivar on disease}

Fusarium head blight (FHB) incidence and DS differed among cultivars and years, often significantly (Table 4). Differences were not usually consistent. In 2011, there was no FHB on cvs Kandela, Łagwa or Ostka Smolicka, and the most FHB was on cvs Parabola (11.5\%) and Tybalt (9.5\%). There was more FHB in 2012 and 2013. In 2012, the least FHB incidence occurred on cv. Trappe (7\%) and the most on cvs Bombona, Izera, Kandela, Katoda, KWS Torridon, Tybalt, and Waluta (10.5-14\%). In 2013, a low FHB incidence (6.5-10\%) was observed on a few cultivars including Arabella (6.5\%) and most occurred again on cv. Tybal (25.5\%). There was strong positive correlation between FHB incidence and DS $(r=-0.925, \mathrm{p} \leq 0.001)$.

\section{Effects of weather on disease}

In 2011, 2012, and 2013, FHB incidence and DS were not correlated with the June-July temperatures. Fusarium head blight incidence was not correlated with total rainfall. Only DS was correlated, at a low level, with July rainfall $(r=0.516-0.657, \mathrm{p} \leq 0.001)$. June rainfall was least in 2011 and 2013 but was associated with lower average FHB and DS values only in 2011.

\section{Colonisation of kernels by Fusarium spp.}

Cultivar affected the colonisation of kernels by Fusarium spp. There were significant differences in proportions of FDK in cultivars in each of the years of the study (Table 5). Averaged over three years, FDK ranged from $13.0 \%$ (cv. Izera) to $35.3 \%$ (cv. Tybalt). In cvs Katoda, KWS Torridon, Monsun, Parabola, Radocha, SMH 87, Struna, Trappe, and Tybalt, the high average (2011-2013) FDK (20.0-35.3\%) was associated with a high average FHB incidence (10.0-16.3\%). In cvs Arabella, Izera, and Kandela, the lower average (2011-2013) FDK (13.0-16.8\%) was associated with a lower average FHB incidence (6.7-9.5\%). The average FDK of all cultivars was similar in all the years of the study. There was a weak, but significant, positive correlation between proportion of FDK and FHB incidence $(r=0.594, \mathrm{p} \leq 0.001)$ and a stronger positive correlation between proportion of FDK and DS $(r=0.733$, $\mathrm{p} \leq 0.001)$. There was a weak, but significant, negative correlation between total rainfall in July and the proportion of $\operatorname{FDK}(r=-0.636, \mathrm{p} \leq 0.001)$.

\section{Fungal species in kernels}

Cultivar significantly affected colonisation of kernels by individual Fusarium species ( $\chi^{2}$ test, $\left.\mathrm{p} \leq 0.05\right)$. Fusarium poae was the Fusarium species isolated most often (Table 6). It was frequent each year, on each cultivar. It colonised $6.0 \%$ of the kernels, on average, but up to $12.0 \%$ on cv. Tybalt. Other Fusarium species occurred less frequently: F. avenaceum (G. avenacea) in 5.6\% of kernels, on average, F. culmorum in 5.3\%, F. tricinctum (G. tricincta) in $2.8 \%$, F. graminearum (G. zeae) in $1.5 \%$, and F. sporotrichioides in $1.2 \%$ of kernels. Fusarium equiseti (G. intricans) was the rarest. Wheat kernels were also colonised by other fungi; the most common were Alternaria alternata, Arthrinium phaeospermum, and Epicoccum nigrum.

\section{Yield}

The total grain yield or thousand-kernel weight (TKW) was not affected by FHB incidence. There was no correlation between average yield of individual cultivars or average TKW in 2011-2013, and average FHB incidence, DS or proportion of FDK. Average grain yield of individual cultivars in 2011-2013 ranged from $6.09 \mathrm{t} \cdot \mathrm{ha}^{-1}$ (cv. SMH 87 ) to $9.41 \mathrm{t} \cdot \mathrm{ha}^{-1}$ (cv. Trappe) (Table 7). The range of the average TKW was from $44.8 \mathrm{~g}$ (cv. Trappe) to $56.0 \mathrm{~g}$ (cv. Parabola). There was a weak, but significant, negative correlation between average yield of individual cultivars in 2011-2013 and TKW $(r=-0.639, \mathrm{p} \leq 0.001)$. The highest yields, in cvs Trappe $\left(9.41 \mathrm{t} \cdot \mathrm{ha}^{-1}\right)$ and KWS Torridon $\left(9.32 \mathrm{t} \cdot \mathrm{ha}^{-1}\right)$, were associated with the lowest TKW $(44.8 \mathrm{~g}$ and $45.9 \mathrm{~g}$, respectively) (Table 8 ). The lowest yield, in cv. SMH $87\left(6.09 \mathrm{t} \cdot \mathrm{ha}^{-1}\right)$, was associated with high TKW (53.2 g), and high FHB incidence (13.5\%) and DS (5.2\%).

\section{Cultivar response to Fusarium infection}

All cultivars expressed 'moderate resistance' when assessed according to the FHB incidence (Table 8). Cultivars 
Table 4. Fusarium head blight (FHB) incidence and disease severity (DS) on different cultivars of spring wheat at Lisewo, in 2011-2013

\begin{tabular}{|c|c|c|c|c|c|c|c|c|}
\hline \multirow{2}{*}{ Cultivar } & \multicolumn{4}{|c|}{ FHB [\%] } & \multicolumn{4}{|c|}{ DS [\%] } \\
\hline & 2011 & 2012 & 2013 & 2011-2013 & 2011 & 2012 & 2013 & 2011-2013 \\
\hline Arabella & - & $10.0^{\mathrm{a}, \mathrm{b}, \mathrm{c}, \mathrm{d}}$ & $6.5^{\mathrm{f}}$ & $18.3^{\mathrm{b}, \mathrm{c}, \mathrm{d}, \mathrm{e}}$ & - & $4.1^{b, c, d}$ & $2.3^{\mathrm{i}}$ & $13.2^{c, d, e, f, g}$ \\
\hline Bombona & $5.0^{\mathrm{b}, \mathrm{c}}$ & $12.5^{\mathrm{a}, \mathrm{b}}$ & $10.0^{\mathrm{c}, \mathrm{d}, \mathrm{e}, \mathrm{f}}$ & $9.2^{b, c, d}$ & $2.3^{a, b, c}$ & $5.5^{\mathrm{a}, \mathrm{b}}$ & $4.1^{\mathrm{e}, \mathrm{f}, \mathrm{g}, \mathrm{h}}$ & $4.0^{\mathrm{c}, \mathrm{d}, \mathrm{e}, \mathrm{f}, \mathrm{g}}$ \\
\hline Hewilla & $4.0^{c}$ & $8.0^{\mathrm{c}, \mathrm{d}}$ & $10.0^{\mathrm{c}, \mathrm{d}, \mathrm{e}, \mathrm{f}}$ & $7.3^{\mathrm{c}, \mathrm{d}, \mathrm{e}, \mathrm{f}}$ & $2.2^{\mathrm{b}, \mathrm{c}}$ & $3.4^{\mathrm{c}, \mathrm{d}}$ & $3.7^{\mathrm{f}, \mathrm{g}, \mathrm{h}, \mathrm{i}}$ & $3.1^{\mathrm{d}, \mathrm{e}, \mathrm{f}, \mathrm{g}}$ \\
\hline Izera & - & $11.5^{\mathrm{a}, \mathrm{b}, \mathrm{c}}$ & $7.5^{\mathrm{e}, \mathrm{f}}$ & $19.5^{a, b, c}$ & - & $4.6^{\mathrm{a}, \mathrm{b}, \mathrm{c}, \mathrm{d}}$ & $2.6^{\mathrm{h}, \mathrm{i}}$ & $13.6^{\mathrm{c}, \mathrm{d}, \mathrm{e}, \mathrm{f}, \mathrm{g}}$ \\
\hline Kandela & $0.0^{\mathrm{d}}$ & $11.0^{\mathrm{a}, \mathrm{b}, \mathrm{c}}$ & $9.0^{\mathrm{d}, \mathrm{e}, \mathrm{f}}$ & $6.7^{\mathrm{d}, \mathrm{e}, \mathrm{f}}$ & $0.0^{c}$ & $4.7^{\mathrm{a}, \mathrm{b}, \mathrm{c}}$ & $3.4^{g, h, i}$ & $2.7^{\mathrm{f}, \mathrm{g}}$ \\
\hline Katoda & $7.5^{\mathrm{a}, \mathrm{b}, \mathrm{c}}$ & $11.0^{\mathrm{a}, \mathrm{b}, \mathrm{c}}$ & $13.0^{\mathrm{b}, \mathrm{c}, \mathrm{d}}$ & $10.5^{\mathrm{a}, \mathrm{b}, \mathrm{c}}$ & $4.2^{\mathrm{a}, \mathrm{b}}$ & $4.6^{\mathrm{a}, \mathrm{b}, \mathrm{c}, \mathrm{d}}$ & $4.7^{\mathrm{d}, \mathrm{e}, \mathrm{f}, \mathrm{g}}$ & $4.5^{\mathrm{b}, \mathrm{c}, \mathrm{d}, \mathrm{e}, \mathrm{f}}$ \\
\hline KWS Torridon & - & $10.5^{\mathrm{a}, \mathrm{b}, \mathrm{c}, \mathrm{d}}$ & $13.0^{\mathrm{b}, \mathrm{c}, \mathrm{d}}$ & ${ }^{1} 11.8^{\mathrm{a}, \mathrm{b}, \mathrm{c}}$ & - & $4.4^{\mathrm{a}, \mathrm{b}, \mathrm{c}, \mathrm{d}}$ & $5.3^{c, d, e}$ & ${ }^{1} 4.9^{\mathrm{a}, \mathrm{b}, \mathrm{c}, \mathrm{d}}$ \\
\hline Łagwa & $0.0^{\mathrm{d}}$ & $7.5^{\mathrm{c}, \mathrm{d}}$ & $8.5^{\mathrm{d}, \mathrm{e}, \mathrm{f}}$ & $5.3^{\mathrm{f}}$ & $0.0^{c}$ & $5.5^{\mathrm{a}, \mathrm{b}}$ & $3.0^{\mathrm{h}, \mathrm{i}}$ & $2.8^{\mathrm{e}, \mathrm{f}, \mathrm{g}}$ \\
\hline Monsun & - & - & $11.0^{\mathrm{b}, \mathrm{c}, \mathrm{d}, \mathrm{e}, \mathrm{f}}$ & ${ }^{2} 11.0^{\mathrm{a}, \mathrm{b}, \mathrm{c}}$ & - & - & $3.6^{\mathrm{f}, \mathrm{g}, \mathrm{g}, \mathrm{i}}$ & $23.6^{\mathrm{c}, \mathrm{d}, \mathrm{e}, \mathrm{f}, \mathrm{g}}$ \\
\hline Nawra & $9.0^{a, b}$ & - & - & $29.0^{\mathrm{b}, \mathrm{c}, \mathrm{d}}$ & $5.0^{\mathrm{a}, \mathrm{b}}$ & - & - & $25.0^{\mathrm{a}, \mathrm{b}, \mathrm{c}, \mathrm{d}}$ \\
\hline Ostka Smolicka & $0.0^{\mathrm{d}}$ & $8.0^{\mathrm{c}, \mathrm{d}}$ & $9.0^{\mathrm{d}, \mathrm{e}, \mathrm{f}}$ & $5.7^{\mathrm{e}, \mathrm{f}}$ & $0.0^{c}$ & $3.2^{\mathrm{c}, \mathrm{d}}$ & $3.4^{g, h, i}$ & $2.2^{\mathrm{g}}$ \\
\hline Parabola & $11.5^{\mathrm{a}}$ & - & $15.5^{b, c}$ & ${ }^{1} 13.5^{\mathrm{a}, \mathrm{b}}$ & $6.4^{\mathrm{a}}$ & - & $6.2^{\mathrm{b}, \mathrm{c}}$ & ${ }^{1} 6.3^{a, b}$ \\
\hline Radocha & - & $9.0^{\mathrm{b}, c, \mathrm{~d}}$ & $14.0^{\mathrm{b}, \mathrm{c}, \mathrm{d}}$ & ${ }^{1} 11.5^{\mathrm{a}, \mathrm{b}, \mathrm{c}}$ & - & $3.8^{\mathrm{b}, \mathrm{c}, \mathrm{d}}$ & $5.8^{b, c, d}$ & ${ }^{1} 4.8^{\mathrm{b}, \mathrm{c}, \mathrm{d}, \mathrm{e}}$ \\
\hline SMH 87 & - & - & $13.5^{\mathrm{b}, \mathrm{c}, \mathrm{d}}$ & ${ }^{2} 13.5^{\mathrm{a}, \mathrm{b}}$ & - & - & $5.2^{\mathrm{c}, \mathrm{d}, \mathrm{e}}$ & $25.2^{a, b, c}$ \\
\hline Struna & - & - & $10.0^{c, d, e, f}$ & ${ }^{2} 10.0^{a, b, c}$ & - & - & $4.0^{\mathrm{e}, \mathrm{f}, \mathrm{g}, \mathrm{h}}$ & ${ }^{2} 4.0^{\mathrm{c}, \mathrm{d}, \mathrm{e}, \mathrm{f}, \mathrm{g}}$ \\
\hline Trappe & - & $7.0^{\mathrm{d}}$ & $16.5^{\mathrm{b}}$ & ${ }^{1} 11.8^{\mathrm{a}, \mathrm{b}, \mathrm{c}}$ & - & $2.9^{\mathrm{d}}$ & $7.0^{\mathrm{b}}$ & $15.0^{\mathrm{a}, \mathrm{b}, \mathrm{c}, \mathrm{d}}$ \\
\hline Tybalt & $9.5^{\mathrm{a}}$ & $14.0^{\mathrm{a}}$ & $25.5^{\mathrm{a}}$ & $16.3^{a}$ & $5.1^{\mathrm{a}, \mathrm{b}}$ & $6.1^{\mathrm{a}}$ & $9.6^{\mathrm{a}}$ & $6.9^{\mathrm{a}}$ \\
\hline Waluta & $4.0^{c}$ & $11.0^{\mathrm{a}, \mathrm{b}, \mathrm{c}}$ & $12.0^{\mathrm{b}, \mathrm{c}, \mathrm{d}, \mathrm{e}}$ & $9.0^{b, c, d}$ & $2.1^{\mathrm{b}, \mathrm{c}}$ & $4.6^{\mathrm{a}, \mathrm{b}, \mathrm{c}, \mathrm{d}}$ & $5.0^{\mathrm{c}, \mathrm{d}, \mathrm{e}, \mathrm{f}}$ & $3.9 c, d, e, f, g$ \\
\hline Average & 5.1 & 10.1 & 12.0 & 10.0 & 2.7 & 4.4 & 4.6 & 4.2 \\
\hline \multicolumn{5}{|c|}{ Least significant difference (LSD) at $p=0.05$} & 4.13 & 1.78 & 1.57 & 2.02 \\
\hline
\end{tabular}

${ }^{1}$ data from two years

${ }^{2}$ data from one year

A different letters in a column indicates significant difference according to one-way ANOVA at $p \leq 0.05$

Table 5. Proportion (\%) of Fusarium damaged kernels (FDK) in different cultivars of spring wheat at Lisewo, in 2011-2013

\begin{tabular}{lcccc}
\hline \multicolumn{1}{c}{ Cultivar } & 2011 & 2012 & 2013 & $2011-2013$ \\
\hline Arabella & - & $17.0^{\mathrm{f}}$ & $13.0^{\mathrm{g}, \mathrm{h}}$ & ${ }^{\mathrm{f}} 15.0^{\mathrm{i}, \mathrm{j}}$ \\
Bombona & $22.0^{\mathrm{c}, \mathrm{d}}$ & $22.0^{\mathrm{c}, \mathrm{d}, \mathrm{e}, \mathrm{f}}$ & $21.0^{\mathrm{c}, \mathrm{d}, \mathrm{e}, \mathrm{f}}$ & $21.7^{\mathrm{e}, \mathrm{f}, \mathrm{g}}$ \\
Hewilla & $31.0^{\mathrm{a}, \mathrm{b}}$ & $20.0^{\mathrm{e}, \mathrm{f}}$ & $22.0^{\mathrm{b}, \mathrm{c}, \mathrm{d}, \mathrm{e}, \mathrm{f}}$ & $24.3^{\mathrm{c}, \mathrm{d}, \mathrm{e}}$ \\
Izera & - & $16.0^{\mathrm{f}}$ & $10.0^{\mathrm{h}}$ & $113.0^{\mathrm{j}}$ \\
Kandela & $16.5^{\mathrm{d}}$ & $18.0^{\mathrm{e}, \mathrm{f}}$ & $16.0^{\mathrm{f}, \mathrm{g}}$ & $16.8^{\mathrm{h}, \mathrm{i}}$ \\
Katoda & $35.0^{\mathrm{a}}$ & $30.0^{\mathrm{a}, \mathrm{b}}$ & $27.0^{\mathrm{b}, \mathrm{c}}$ & $30.7^{\mathrm{b}}$ \\
KWS Torridon & - & $24.0^{\mathrm{b}, \mathrm{c}, \mathrm{d}, \mathrm{e}}$ & $22.0^{\mathrm{b}, \mathrm{d}, \mathrm{d}, \mathrm{e}, \mathrm{f}}$ & $123.0^{\mathrm{d}, \mathrm{e}, \mathrm{f}}$ \\
Eagwa & $36.0^{\mathrm{a}}$ & $21.0^{\mathrm{d}, \mathrm{e}, \mathrm{f}}$ & $10.0^{\mathrm{h}}$ & $22.3^{\mathrm{d}, \mathrm{e}, \mathrm{f}, \mathrm{g}}$ \\
Monsun & - & - & $20.0^{\mathrm{d}, \mathrm{e}, \mathrm{f}}$ & $220.0^{\mathrm{f}, \mathrm{g}, \mathrm{h}}$ \\
Nawra & $25.5^{\mathrm{b}, \mathrm{c}}$ & - & - & $225.5^{\mathrm{c}, \mathrm{d}, \mathrm{e}}$ \\
Ostka Smolicka & $26.0^{\mathrm{b}, \mathrm{c}}$ & $20.0^{\mathrm{e}, \mathrm{f}}$ & $19.3^{\mathrm{f}, \mathrm{g}, \mathrm{h}}$ \\
Parabola & $29.0^{\mathrm{a}, \mathrm{b}}$ & - & $12.0^{\mathrm{g}, \mathrm{h}}$ & $127.5^{\mathrm{b}, \mathrm{c}}$ \\
Radocha & - & $27.0^{\mathrm{a}, \mathrm{b}, \mathrm{c}, \mathrm{d}}$ & $26.0^{\mathrm{b}, \mathrm{c}, \mathrm{d}}$ & $22.0^{\mathrm{c}, \mathrm{d}}$ \\
SMH 87 & - & - & $25.0^{\mathrm{b}, \mathrm{c}, \mathrm{d}, \mathrm{e}}$ & $223.0^{\mathrm{d}, \mathrm{e}, \mathrm{f}}$ \\
Struna & - & - & $23.0^{\mathrm{b}, \mathrm{c}, \mathrm{d}, \mathrm{e}}$ & $219.0^{\mathrm{g}, \mathrm{h}}$ \\
Trappe & - & $19.0^{\mathrm{e}, \mathrm{f}}$ & $128.0^{\mathrm{b}, \mathrm{c}}$ \\
Tybalt & $34.0^{\mathrm{a}}$ & $28.0^{\mathrm{a}, \mathrm{b}, \mathrm{c}}$ & $28.0^{\mathrm{b}}$ & $35.3^{\mathrm{a}}$ \\
Waluta & $29.5^{\mathrm{a}, \mathrm{b}}$ & $32.0^{\mathrm{a}}$ & $40.0^{\mathrm{a}}$ & $24.5^{\mathrm{c}, \mathrm{d}, \mathrm{e}}$ \\
Average & 28.5 & $22.0^{\mathrm{c}, \mathrm{d}, \mathrm{e}, \mathrm{f}}$ & $22.0^{\mathrm{b}, \mathrm{c}, \mathrm{d}, \mathrm{e}, \mathrm{f}}$ & 23.1 \\
\hline
\end{tabular}

${ }^{1}$ data from two years

${ }^{2}$ data from one year

A different letters in a column indicates significant difference according to $\chi^{2}$ test at $p \leq 0.05$ 
ęnโำM

†ाeq $\mathcal{L}_{\mathrm{L}}$

əddexi

ind

zeunins

¿ $\angle 8$ HWS

¿ечрореу

${ }_{\text {I }}$ [oquix $_{\mathrm{d}}$

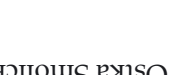

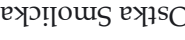

(2)

${ }_{\text {¿.JMen }}$

zunsuow

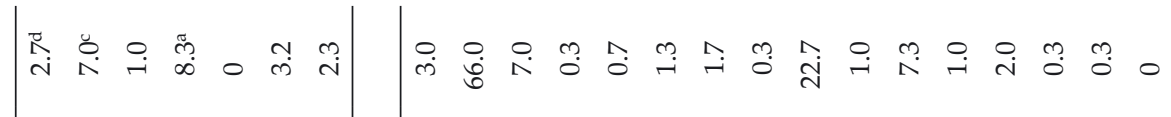
药

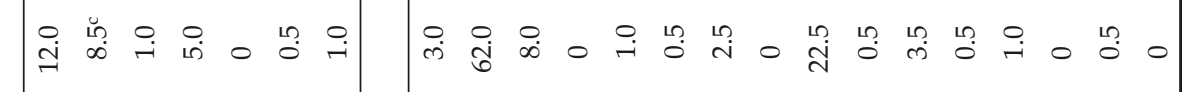
ம்

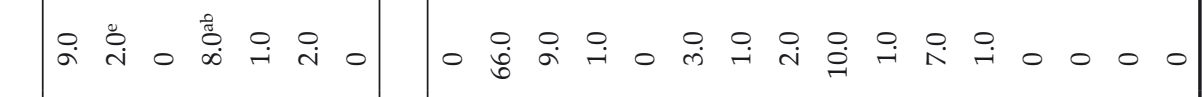

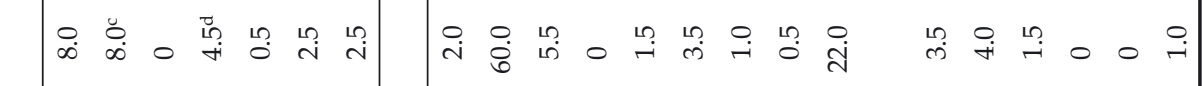

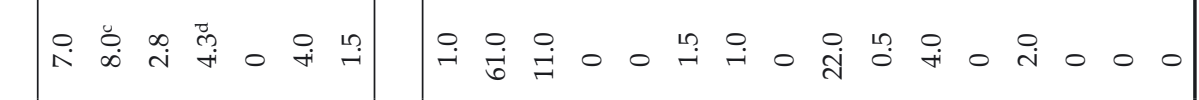

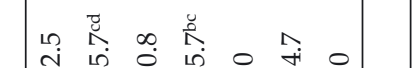

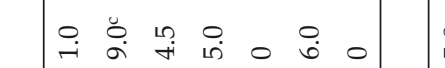

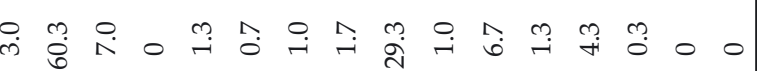
in

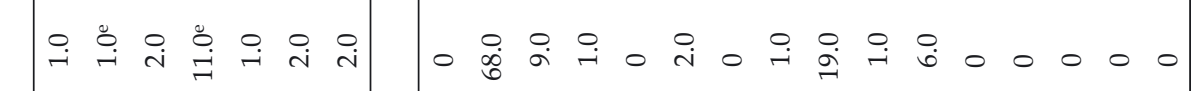
ем.8е 究

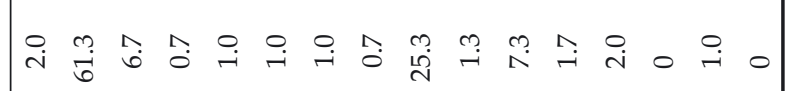

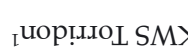
ำ 绾 ерояех ерәрие

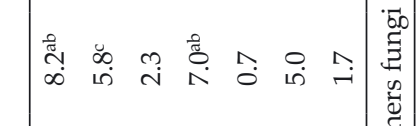

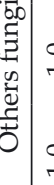

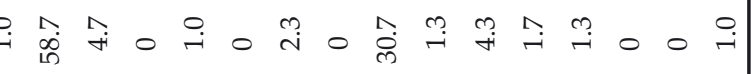

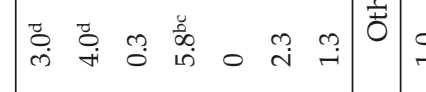

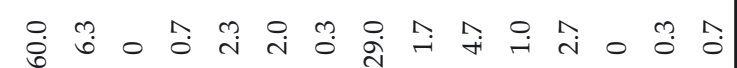
ñ ${ }_{1}^{\text {exəzZI }}$ ${ }^{\mathrm{E}}[\mathrm{I} ! \mathrm{M} ә \mathrm{H}$

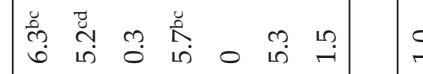
암 euoquog

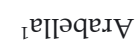

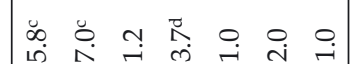
군 서

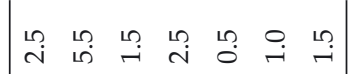
엉 苟

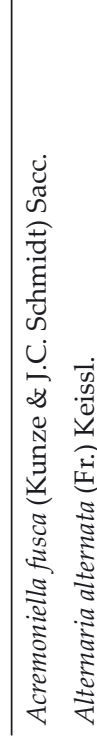

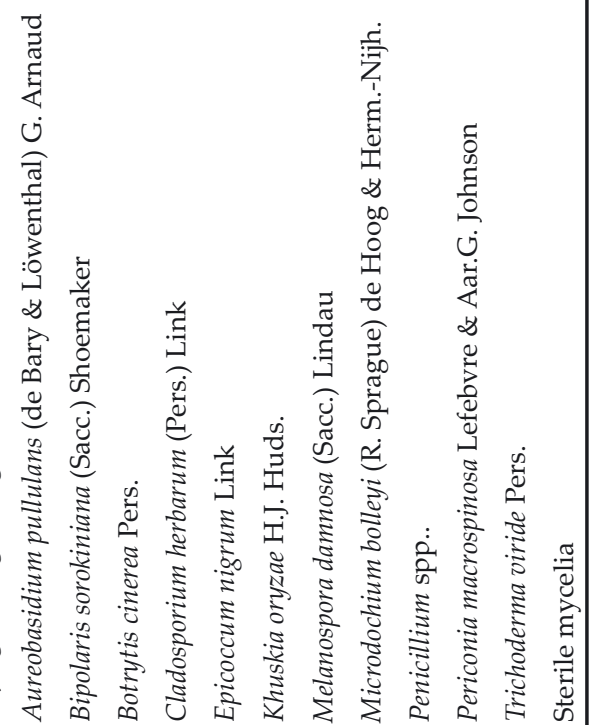

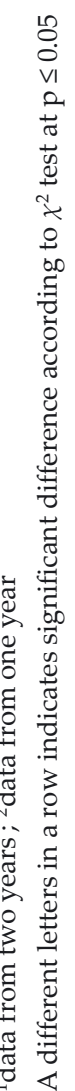


Table 7. Grain yield and thousand kernel weight (TKW) in different cultivars of spring wheat at Lisewo, in 2011-2013 (according to the Experimental Station for Variety Testing in Lisewo Malborskie)

\begin{tabular}{|c|c|c|c|c|c|c|c|c|}
\hline \multirow[b]{2}{*}{ Cultivar } & \multicolumn{4}{|c|}{ Yield $\left[t \cdot h^{-1}\right]$} & \multicolumn{4}{|c|}{ TKW [g] } \\
\hline & 2011 & 2012 & 2013 & $\begin{array}{c}\text { average } \\
2011-2013\end{array}$ & 2011 & 2012 & 2013 & $\begin{array}{c}\text { average } \\
2011-2013\end{array}$ \\
\hline Bombona & 8.04 & 9.29 & 9.22 & 8.85 & 49.0 & 46.6 & 42.4 & 46.0 \\
\hline Hewilla & 8.21 & 8.81 & 8.77 & 8.59 & 54.0 & 50.5 & 46.6 & 50.4 \\
\hline Izera & - & 8.91 & 8.95 & 8.93 & - & 48.6 & 43.9 & 46.3 \\
\hline Kandela & 8.04 & 9.29 & 9.22 & 8.85 & 52.4 & 51.7 & 47.1 & 50.4 \\
\hline Katoda & 8.46 & 9.10 & 8.68 & 8.74 & 53.6 & 48.7 & 47.1 & 49.8 \\
\hline KWS Torridon & - & 9.87 & 8.77 & 9.32 & - & 45.3 & 46.4 & 45.9 \\
\hline Łagwa & 8.12 & 8.62 & 9.22 & 8.65 & 53.8 & 49.7 & 50.3 & 51.3 \\
\hline Ostka Smolicka & 8.80 & 8.62 & 8.41 & 8.61 & 54.4 & 47.6 & 47.3 & 49.8 \\
\hline Parabola & 8.12 & - & - & 8.12 & 56.0 & - & - & 56.0 \\
\hline Radocha & 8.12 & 8.14 & - & 8.13 & 55.2 & 51.7 & - & 53.5 \\
\hline SMH 87 & 6.09 & - & - & 6.09 & 53.2 & - & - & 53.2 \\
\hline Struna & - & - & 9.04 & 9.04 & - & - & 45.5 & 45.5 \\
\hline Trappe & 8.97 & 10.15 & 9.13 & 9.41 & 49.6 & 44.5 & 40.3 & 44.8 \\
\hline Tybalt & 8.63 & 9.48 & 9.22 & 9.11 & 54.0 & 50.0 & 47.2 & 50.4 \\
\hline Waluta & 7.87 & 8.43 & 8.50 & 8.26 & 51.2 & 48.5 & 46.5 & 48.7 \\
\hline
\end{tabular}

Table 8. Resistance category of spring wheat cultivars determined from assessments, in 2011-2013

\begin{tabular}{|c|c|c|c|c|}
\hline \multirow{2}{*}{ Cultivar } & \multicolumn{2}{|c|}{ Cultivar response } & \multirow{2}{*}{ FHB [\%] } & \multirow{2}{*}{ FDK [\%] } \\
\hline & based on FHB incidence & based on FDK & & \\
\hline Arabella & moderately resistant & moderately susceptible & 8.3 & 15.0 \\
\hline Bombona & moderately resistant & susceptible & 9.2 & 21.7 \\
\hline Hewilla & moderately resistant & susceptible & 7.3 & 24.3 \\
\hline Izera & moderately resistant & moderately susceptible & 9.5 & 13.0 \\
\hline Kandela & moderately resistant & moderately susceptible & 6.7 & 16.8 \\
\hline Katoda & moderately resistant & susceptible & 10.5 & 30.7 \\
\hline KWS Torridon & moderately resistant & susceptible & 11.8 & 23.0 \\
\hline Łagwa & moderately resistant & susceptible & 5.3 & 22.3 \\
\hline Monsun & moderately resistant & moderately susceptible & 11.0 & 20.0 \\
\hline Nawra & moderately resistant & susceptible & 9.0 & 25.5 \\
\hline Ostka Smolicka & moderately resistant & moderately susceptible & 5.7 & 19.3 \\
\hline Parabola & moderately resistant & susceptible & 13.5 & 27.5 \\
\hline Radocha & moderately resistant & susceptible & 11.5 & 26.0 \\
\hline SMH 87 & moderately resistant & susceptible & 13.5 & 23.0 \\
\hline Struna & moderately resistant & moderately susceptible & 10.0 & 19.0 \\
\hline Trappe & moderately resistant & susceptible & 11.8 & 28.0 \\
\hline Tybalt & moderately resistant & susceptible & 16.3 & 35.3 \\
\hline Waluta & moderately resistant & susceptible & 9.0 & 24.5 \\
\hline
\end{tabular}

FHB - Fusarium head blight; FDK - Fusarium-colonised kernels 
Arabella, Izera, Kandela, Monsun, Ostka Smolicka, and Struna expressed 'moderate susceptibility' and cvs Bombona, Hewilla, Katoda, KWS Torridon, Łagwa, Nawra, Parabola, Radocha, SMH 87, Trappe, Tybalt, and Waluta expressed 'susceptibility' when assessed by proportion of FDK. Cultivars differed in their levels of 'moderate resistance', 'moderate susceptibility', and 'susceptibility'.

\section{Discussion}

Growing wheat cultivars with greater resistance to FHB is the most promising strategy for disease control. Fusarium head blight resistance is, however, a complex trait. To date, sources of resistance conferring complete resistance to FHB have not been identified in wheat. Resistance to FHB has been shown to be under the control of a few major QTLs operating together with unknown numbers of minor genes (Buerstmayr et al. 2009; Kollers et al. 2013).

Reported sources of FHB resistance in spring wheat include a few landraces, 'Sumai 3 ' and its derivatives from China, 'Nobeoka Bozu' and 'Sin Chunaga' and its relatives from Japan, and 'Frontana' and 'Encruzilhada' from Brazil (Mesterhazy 1987; Liu and Wang 1991; Ban and Suenaga 2000; Yu et al. 2006).

Most success has been made in transferring FHB resistance from the Chinese wheat cultivar Sumai 3 (Anderson et al. 2001, 2007; Rudd et al. 2001). Cultivar Sumai 3 has the FHB resistance gene Fhb1, which has been extensively used in breeding programs as a major source of partial resistance to FHB. It showed a major effect on Type II resistance across different genetic backgrounds and environments. Other QTLs for FHB resistance have exhibited minor effects and their expression varied significantly.

Heavy use of narrow FHB resistance sources may increase selection pressure on the pathogens to erode the efficacy of the resistance genes involved. Thus, identification and characterisation of additional sources of resistance are important for enhancing the level of resistance and for introducing genetic variation to the breeding materials. Evaluation of FHB resistance in local cultivars of spring wheat may provide: (i) good lines for local breeding programmes and (ii) selected resistant cultivars for commercial farming aimed at production of mycotoxinfree grain in integrated FHB management systems based on forecasting models.

Spring wheat cultivars in Europe have the highest levels of resistance to FHB when compared with cultivars from South America or Asia (Zhang et al. 2008). Polish cultivars and foreign cultivars/lines have been continuously screened for FHB resistance (Góral 2005; Wiśniewska and Kowalczyk 2005; Lenc and Sadowski 2011; Góral and Walentyn-Góral 2014). Research has also concentrated on how the previous crop and weather conditions affect infection by Fusarium, and on concentrations of mycotoxins in grain (Sadowski et al. 2011; Góral et al. 2012). The most resistant genotypes, with acceptable agronomic characters, are used in farming and breeding, often despite the failure to identify their sources of resistance.

The results reported here showed significant differences in FHB incidence among spring wheat cultivars included in the Polish National List of Agricultural Plant
Varieties, in natural non-epidemic conditions, in northern Poland, from 2011 to 2013 . The official catalogues of varieties describe the $\mathrm{FHB}$ resistance of particular cultivars as average (cv. Łagwa), moderate (cvs Arabella, Hewilla, Kandela, Katoda, KWS Torridon, Monsun, Nawra, Ostka Smolicka, Parabola, Radocha, SMH 87, Trappe, Tybalt, and Waluta) or very good (cvs Bombona, Izera, and Struna). The average FHB severity, which in Lisewo ranged from $2.2-6.9 \%$, is consistent with the general resistance to Fusarium spp., which would have contributed to the 2011-2013, non-epidemic situatiolt should be noted, that there were significant differences within this 'general resistance'. Only cv. Tybalt had FHB severity (DS) approaching $7 \%$, which is the lower limit for light epidemics (Del Ponte et al. 2005). The cultivars compared were being recommended for commercial production in northern Poland in 2014 (Anonymous 2014). Cultivars Bombona, Hewilla, Kandela, Katoda, Łagwa, Monsun, Nawra Ostka Smolicka, Parabola, Trappe, and Waluta were also being used for the production of seeds. Each cultivar was on an area of 51-445 ha (Góral and Walentyn-Góral 2014). Choosing more susceptible cultivars would be associated with a greater risk of Fusarium mycotoxin contamination, a decreased germination rate, and poor seedling growth (Gilbert and Tekauz 1995). The use of infected grain can, by addition of virulent toxigenic biotypes of fungi, additionally enrich the complex of soil phytopathogens associated with seedling blight.

Fusarium head blight (FHB) is a major fungal disease in durum wheat. Fewer sources of resistance have been found in tetraploid durum wheat than in hexaploid wheat (Rudd et al. 2001; Oliver et al. 2008). There was high FHB incidence $(13.5 \%)$ and disease severity $(5.2 \%)$ in the single durum wheat cultivar (SMH 87) that we tested. The high FHB incidence was associated with the smallest grain yield $\left(6.09 \mathrm{t} \cdot \mathrm{ha}^{-1}\right)$. Durum wheat is said to be susceptible mostly to F. graminearum (G. zeae). It was suggested by Lionetti et al. (2015) that content and composition of cell wall polymers affect susceptibility to the wall-degrading enzymes produced by F. graminearum during infection, which affects the outcome of host-pathogen interactions. This fungus was not found in cv. SMH 87. Infected kernels were colonised mostly by G. avenacea.

Resistance of Type I (to initial infection), Type II (to spread within the head) and Type IV (to kernel infection) to FHB, were assessed in this study. Types I and II were assessed from FHB incidence and DS on developing heads, and Type IV from the proportion of FDK as suggested by Mesterhazy et al. (1999). Type III resistance (resistance to DON accumulation) is also said to be an important component of FHB resistance (Miller et al. 1985; Snijders and Perkowski 1990) but was not included in our study. Fusarium head blight incidence is not always correlated with DON concentration (Mesterházy et al. 1999; Bai et al. 2001; Koch et al. 2006; Brennan et al. 2007; Lehoczki-Krsjak et al. 2010; Wegulo et al. 2011; Gromadzka et al. 2012). It was assumed here, that cultivars with low kernel colonisation by Fusarium spp. had low concentrations of mycotoxins and stable FHB resistance (Snijders and Krechting 1992; Bai et al. 2001). 
The assessments based on FHB incidence and DS confirmed general 'moderate FHB resistance' of the cultivars included in the study. The category 'moderately resistant' is based, however, on a wide range (5-25\%) of FHB incidence values. On this basis, expression of resistance within the 'moderate resistance' category, was greatest in cvs Arabella, Bombona, Hewilla, Izera, Kandela, Łagwa, Nawra, Ostka Smolicka, Struna, and Waluta (FHB = 5.3$10.0 \%$, DS $=2.2-5.0 \%$ ). Less resistance in this 'moderate resistance' category was expressed by cvs Katoda, KWS Torridon, Monsun, Parabola, Radocha, SMH 87, Trappe, and Tybalt $(\mathrm{FHB}=10.5-16.3 \%, \mathrm{DS}=3.6-6.9 \%)$. On the basis of the proportion of FDK, the cultivars were categorised as 'moderately susceptible/susceptible', i.e. with lower levels of resistance. These assessments of cultivar response to Fusarium infection in the field differ from the results of Zhang et al. (2008). They found that European cultivars of spring wheat with 'moderate resistance' in the field displayed higher levels of resistance based on the proportion of FDK.

There was a positive correlation between FHB incidence and the proportion of FDK. In more resistant cultivars, there was less kernel colonisation. An explanation is that limited fungal colonisation of kernels in resistant cultivars results from membrane-based tolerance of trichothecene mycotoxins (Snijders and Krechting 1992). However, a lack of significant correlation between FHB incidence and the proportion of FDK has also been reported, and attributed to two different sources of resistance (Sneller et al. 2012).

None of the cultivars in the present study was highly resistant to FHB. This is partly in agreement with Góral and Walentyn-Góral (2014) who found statistically significant variability in FHB resistance and a wide range of FHB incidences among 25 spring wheat cultivars from the Polish National List and 35 cultivars/lines from a collection of resistant forms over a three-year study (2010-2012). They found moderate FHB resistance in cvs Bombona, Łagwa, Monsun, Ostka Smolicka, Trappe, and Waluta, and greater resistance in cvs Hewilla, Kandela, Katoda, Nawra, and Parabola.

Fusarium poae, F. avenaceum, and F. culmorum were the most frequent Fusarium spp. in spring wheat kernels. They were also the most frequent in a study on winter wheat in Poland (Lenc et al. 2011; Lenc 2015). These species, together with F. graminearum, F. pseudograminearum O’Donnell \& T. Aoki (teleomorph Gibberella coronicola T. Aoki \& O'Donnell), Microdochium nivale (Fr.) Samuels \& I.C. Hallett (teleomorph Monographella nivalis (Schaffnit) E. Müll.), and M. majus (Wollenw.) Glynn \& S.G. Edwards, form the dominant group in FHB populations in Europe, USA, and Canada (Wilcoxon et al. 1988; Gale et al. 2007; Alvarez et al. 2010). Fusarium avenaceum, F. culmorum, and F. poae are more adapted to cooler/wet/humid regions (Doohan et al. 2003), although Xu et al. (2008) also associated $F$. poae with relatively drier and warmer conditions. According to Rohácik and Hudec (2005), a high incidence and density of $F$. poae in warmer places results from its adaptability to agro-environmental conditions during grain formation.
In general terms, F. poae and F. avenaceum are relatively weaker pathogens than F. graminearum and F. culmorum (Wong et al. 1992; Fernandez and Chen 2005; Xu et al. 2007). Only individual isolates of $F$. poae are strongly aggressive (comparable with F. culmorum and F. graminearum) (Brennan et al. 2007). It is receiving increased interest because of its toxigenic potential (it produces trichothecenes of types $\mathrm{A}$ and $\mathrm{B}$, aurofusarin, beauvericin, butenolide, culmorin, cyclonerodiol, enniatins, fusarin, and moniliformin) and the human and animal mycotoxicoses it can cause (De Nijs et al. 1996a, b; Thrane et al. 2004; Chełkowski et al. 2007).

Fusarium graminearum was relatively rare in the spring wheat cultivars although its increasing contribution to FHB in Poland has been observed (Czaban et al. 2011; Lenc 2015). In general, F. graminearum favors warmer weather. The increasing significance of $F$. graminearum is related to global warming (higher temperatures in spring and summer) and increased amounts of maize residues and fungal inoculum associated with increased production of maize for grain (Wakuliński and Chełkowski 1993). Fusarium langsethiae Torp and Nirenberg, a species with increasing significance in Poland (Łukanowski et al. 2008; Łukanowski and Sadowski 2008), was not recorded.

The colonisation of kernels by other pathogenic or saprotrophic fungi may increase the likelihood of further deterioration and additional chemical contamination of the grain. The taxa recorded (Table 6) are often known to be secondary invaders, which can cause serious storage mold problems. Alternaria, Aspergillus, and Penicillium spp. are known mycotoxin producers (Steyn 1995). Their presence in grain constitutes a potential additional health risk. Storage of cereals under warm and humid conditions may further increase mycotoxin content even when field infections were only light to moderate. Trichoderma viride, which is antagonistic towards Fusarium spp. with a potential to reduce inoculum development (Inch and Gilbert 2007), occurred sporadically.

The studies were carried out in only one location, but the complexity of resistance mechanisms and significant environment effects on FHB development require screening in a range of environments (Fuentes et al. 2005). The location in northern Poland, however, is in the main target area for spring wheat production because of the weather conditions. In addition, the 'moderately resistant' categorisation of the cultivars chosen for study ensures that they should have some stability under different epidemic conditions. According to Mesterházy (1995), the stability of plant reaction is connected to the resistance level; the most resistant genotypes are the most stable, and the most susceptible ones tend to be unstable.

Weather is usually the main determinant of FHB incidence (Parry et al. 1995; De Wolf et al. 2003; Lemmens et al. 2004; Xu et al. 2008). Epidemics of FHB are associated with multiple inoculation episodes and with coincidental wet periods. Higher humidity favors the growth and sporulation of Fusarium fungi which are spread by wind and rain splashes. Lower humidity helps plants to dry and prevents infection and colonisation. In this study, though, a decreased average proportion of FDK was observed with increased rainfall in July. There was intense 
and prolonged rain in July 2011-2013. Rain intensity, duration, and frequency, as well as the size and velocity of falling drops, affect the splash dispersal of spores and success of infection (Shin et al. 2014). Intense rain may have a decreased effective dispersal by washing off newly-dispersed spores, which therefore, did not contribute to increased Fusarium infection (Penet et al. 2014). Wind will increase primary rain-dispersal distance in a downwind direction and decrease it upwind, although it is accepted that wind-dispersal distances are generally longer than rain dispersal alone, and that pure splash dispersal is mostly local (Yang et al. 1990; Sache 2000). The epidemiology of FHB caused by F. poae and the pathogen's infection biology are less understood then other major FHB patogens (Stenglein 2009).

\section{Conclusions}

Eighteen spring wheat cultivars were all found to be 'moderately resistant' to 'moderately susceptible' to FHB in non-epidemic situations in the field. There were differences within the wide range of resistance/susceptibility categories. The cultivars showing most resistance (Arabella, Izera, Kandela, Monsun, Ostka Smolicka, and Struna) can be recommended for breeding programmes and for commercial farming with the aim of producing mycotoxin-free grain in integrated FHB management systems.

\section{Acknowledgements}

The authors thank the Head of the Experimental Station for Variety Testing in Karżniczka for his cooperation in allowing this research to be carried out on the premises.

This research was supported by the University of Technology and Life Sciences in Bydgoszcz, BS 8/2012 "Pathogenic and nonpathogenic fungi in the natural environment".

\section{References}

Alvarez C.L., Somma S., Moretti A., Fernandez Pinto V. 2010. Aggressiveness of Fusarium graminearum sensu stricto isolates in wheat kernels in Argentina. Journal of Phytopathology 158 (3): 173-181.

Anderson J.A., Stack R.W., Liu S., Waldron B.L., Fjeld A.D., Coyne C., Moreno-Sevilla B., Fetch J.M., Song Q.J., Cregan P.B., Frohberg R.C. 2001. DNA markers for Fusarium head blight resistance QTLs its two wheat populations. Theoretical and Applied Genetics 102 (8): 1164-1168.

Anderson J.A., Chao S., Liu S. 2007. Molecular breeding using a major QTL for Fusarium head blight resistance in wheat. Crop Science 47 (3): 112-119.

Anonymous 2014. Lista Odmian Zalecanych (LOZ) do uprawy na rok $2014 \mathrm{w}$ województwie kujawsko-pomorskim. [List of cultivars recommended for growing in northern Poland in 2014]. Kujawsko-Pomorski Ośrodek Doradztwa Rolniczego w Minikowie, Minikowo, Poland. (in Polish)

Bai G.H., Plattner R., Desjardins A., Kolb F. 2001. Resistance to Fusarium head blight and deoxynivalenol accumulation in wheat. Plant Breeding 120 (1): 1-6.
Ban T., Suenaga K. 2000. Genetic analysis of resistance to Fusarium head blight caused by Fusarium graminearum in Chinese wheat cultivar Sumai 3 and the Japanese cultivar Saikai 165. Euphytica 113 (2): 87-99.

Booth C. 1971. The Genus Fusarium. Commonwealth Mycological Institute, Kew, Surrey, England, 237 pp.

Brennan J.M., Leonard G., Fagan B., Cooke B.M., Ritieni A., Ferracane R., Nicholson P., Simpson D., Thomsett M., Doohan F.M. 2007. Comparison of commercial European wheat cultivars to Fusarium infection of head and seedling tissue. Plant Pathology 56 (1): 55-64.

Buerstmayr H., Ban T., Anderson J.A. 2009. QTL mapping and marker-assisted selection for Fusarium head blight resistance in wheat: a review. Plant Breeding 128 (1): 1-26.

Chełkowski J., Ritieni A., Wiśniewska H., Mulé G., Logrieco A. 2007. Occurrence of toxic hexadepsipeptides in preharvest maize ear rot infected by Fusarium poae in Poland. Journal of Phytopathology 155 (1): 8-12.

Crute I.R., Wit P.J.G.M., Wade M. 1985. Mechanisms by which genetically controlled resistance and virulence influence host colonization by fungal and bacterial parasites. p. 197-309. In: "Mechanism of Resistance to Plant Diseases" (R.S.S. Fraser, ed.). Martinus Nijhoff/Kluwer Academic, Dordrecht, The Netherlands, $473 \mathrm{pp}$.

Czaban J., Wróblewska B., Sułek A., Podolska G. 2011. Colonization of wheat grain by Fusaria in two crop management systems varying in intensity of production technology. Plant Breeding and Seed Science 64: 3-13.

De Nijs M., Rombouts F., Notermans S. 1996a. Fusarium molds and their mycotoxins. Journal of Food Safety 16 (1): 15-58.

De Nijs M., Soentoro P., Delffgou-Van Asch E., Kamphuis H., Rombouts F.M., Notermans S.H.W. 1996b. Fungal infection and presence of deoxynivalenol and zearalenons in cereals grown in the Netherlands. Journal of Food Protection 59 (6): 772-777.

De Wolf E.D., Madden L.V., Lipps P.E. 2003. Risk assessment models for wheat Fusarium head blight epidemics based on within-season weather data. Phytopathology 93 (4): 428-435.

Del Ponte E.M., Fernandes J.M.C., Pavan W. 2005. A risk infection simulation model for Fusarium head blight of wheat. Fitopatologia Brasileira 30 (6): 634-642.

Doohan F.M., Brennan J., Cooke B.M. 2003. Influence of climatic factors on Fusarium species pathogenic to cereals. European Journal of Plant Pathology 109 (7): 755-768.

Fedak G., Gilbert J., Comeau A., Voldeng H., Savard M., Butler G. 2001. Sources of Fusarium head blight resistance in spring wheat. p. 30-35. In: "Proceeding of the Second Canadian Workshop on Fusarium Head Blight" (G. Fedak, A. Choo, eds.), Ottawa, Canada.

Fernandez M.R., Chen Y. 2005. Pathogenicity of Fusarium species on different plant parts of spring wheat under controlled conditions. Plant Disease 89 (2): 164-169.

Fuentes R.G., Mickelson H.R., Busch R.H., Dill-Macky R., Evans C.K., Thompson W.G., Wiersma J.V., Xie W., Dong Y., Anderson J.A. 2005. Resource allocation and cultivar stability in breeding for Fusarium head blight resistance in spring wheat. Crop Science 45 (5): 1965-1972.

Gale L.R., Ward T.J., Balmas V., Kistler H.C. 2007. Population subdivision of Fusarium graminearum sensu stricto in the 
upper Midwestern United States. Phytopathology 97 (11): 1434-1439.

Gilbert J., Tekauz A. 1995. Effects of Fusarium head blight and seed treatment on germination, emergence and seedling vigour of spring wheat. Canadian Journal of Plant Pathology 17 (3): 252-259.

Góral T. 2005. Źródła odporności pszenicy na fuzariozę kłosa powodowaną przez Fusarium culmorum (W. G. Smith) Sacc. [Sources of wheat resistance to Fusarium head blight caused by Fusarium culmorum (W. G. Smith) Sacc.]. Biuletyn IHAR 235: 115-131. (in Polish)

Góral T., Ochodzki P., Walentyn-Góral D., Nielsen L.K., Justesen A.F., Jorgensen L.N. 2012. Wpływ przedplonu oraz warunków pogodowych na porażenie kłosów pszenicy jarej przez grzyby z rodzaju Fusarium oraz zawartość mikotoksyn w ziarnie. [Effect of pre-crop and weather conditions on infection of heads of spring wheat with Fusarium fungi and content of mycotoxins in grain]. Biuletyn IHAR 265: 11-21. (in Polish)

Góral T., Walentyn-Góral D. 2014. Odporność odmian i linii pszenicy jarej na fuzariozę kłosów powodowaną przez grzyb Fusarium culmorum. [Resistance of spring wheat cultivars and lines to Fusarium head blight caused by Fusarium culmorum]. Biuletyn IHAR 271: 3-16. (in Polish)

Grabowski A., Siuda R., Lenc L., Jaroszuk-Ściseł J. 2012a. Effect of the degree of fusariosis on the physical characteristics of individual wheat kernels. International Journal of Food Science and Technology 47 (6): 1122-1129.

Grabowski A., Siuda R., Lenc L., Grundas S. 2012b. Evaluation of single-kernel density of scab-damaged winter wheat. International Agrophysics 26 (2): 129-135.

Gromadzka K., Lenc L., Sadowski Cz., Baturo-Cieśniewska A., Chełkowski J., Goliński P., Bocianowski J. 2012. Effects of fungicidal protection programs on the development of $\mathrm{Fu}$ sarium head blight and the accumulation of mycotoxins in winter wheat. Cereal Research Communications 40 (4): 518-531.

Inch S., Gilbert J. 2007. Effect of Trichoderma harzianum on perithecial production of Gibberella zeae on wheat straw. Biocontrol Science and Technology 17 (5/6): 635-646.

Koch H.J., Pringas C., Maerlaender B. 2006. Evaluation of environmental and management effects on Fusarium head blight infection and deoxynivalenol concentration in the grain of winter wheat. European Journal of Agronomy 24 (4): 357-366.

Kollers S., Rodemann B., Ling J., Korzun V., Ebmeyer E., Argillier O., Hinze M., Plieske J., Kulosa D., Ganal M.W., Röder M. 2013. Whole genome association mapping of Fusarium head blight resistance in European winter wheat (Triticum aestivum L.). PLOS ONE 8 (2): e 57500. DOI: 10.1371/journal.pone.0057500.

Kubo K., Kawada N., Fujita M. 2013. Evaluation of Fusarium head blight resistance in wheat and the development of a new variety by integrating Type I and II Resistance. Japan Agricultural Research Quarterly 47 (1): 9-19.

Kwaśna H., Chełkowski J., Zajkowski P. 1991. Grzyby (Mycota). [Fungi (Mycota)]. Vol. XXII. Polish Academy of Sciences, Warszawa-Kraków, 137 pp. (in Polish)

Lehoczki-Krsjak S., Szavo-Hever A., Toth B., Kotai C., Bartok T., Varga M., Faraday L., Mesterházy A. 2010. Prevention of Fusarium mycotoxin contamination by breeding and fungi- cide application to wheat. Food Additives and Contamination 27 (5): 616-628.

Lemmens M., Buerstmayr H., Krska R., Schuhmacher R. 2004. The effect of inoculation treatment and long-term application of moisture on Fusarium head blight symptoms and deoxynivalenol contamination in wheat grains. European of Journal of Plant Pathology 110 (3): 299-308.

Lenc L. 2015. Fusarium head blight (FHB) and Fusarium populations in grain of winter wheat grown in different cultivation systems. Journal of Plant Protection Research 55 (1): 94-109.

Lenc L., Kuś J., Sadowski Cz. 2011. Fusarium spp. on ears and kernels of winter wheat in different cropping systems. Journal of Research and Applications in Agricultural Engineering 56 (4): 32-36.

Lenc L., Sadowski Cz. 2011. Wrażliwość 16 odmian pszenicy jarej uprawianej na Żuławach i w rejonie Bydgoszczy na fuzariozę kłosów (Fusarium spp.). [The susceptibility of 16 spring wheat cultivars grown in the area Żuławy and in Bydgoszcz on Fusarium head blight (Fusarium spp.)]. Progress in Plant Protection/Postępy w Ochronie Roślin 51 (2): 799-804. (in Polish)

Lionetti V., Giancaspro A., Fabri E., Giove S.L., Reem N., Zabotina O.A., Blanco A., Gadaleta A., Bellincampi D. 2015. Cell wall traits as potential resources to improve resistance of durum wheat against Fusarium graminearum. BioMed Central Plant Biology 15: 6. DOI: 10.1186/s12870-014-0369-1.

Liu Z.Z., Wang Z.Y. 1991. Improved scab resistance in China: Sources of resistance and problems. p. 178-188. In: "Wheat for the Nontraditional Warm Areas" (D.A. Saunders, ed.). International Maize and Wheat Improvement Center (CIMMYT), Mexico, D.F., Mexico.

Łukanowski A., Lenc L., Sadowski Cz. 2008. First report on the occurrence of Fusarium langsethiae isolated from wheat kernels in Poland. Plant Diseases 92 (3): 488.

Łukanowski A., Sadowski Cz. 2008. Fusarium langsethiae on kernels of winter wheat in Poland - occurrence and mycotoxigenic abilities. Cereal Research Communications 36: 453-457.

Mesterházy Á. 1987. Selection of head blight resistant wheats through improved seedling resistance. Plant Breeding 98 (1): 25-36.

Mesterházy Á. 1995. Types and components of resistance to Fusarium head blight of wheat. Plant Breeding 114 (5): 377-386.

Mesterházy Á., Bartók T., Mirocha C.G., Komoróczy R. 1999. Nature of wheat resistance to Fusarium head blight and the role of deoxynivalenol for breeding. Plant Breeding 118 (2): 97-110.

Miedaner T., Perkowski J. 1996. Correlation among Fusarium culmorum head blight resistance, fungal colonization and mycotoxin content in winter rye. Plant Breeding 115 (5): 347-351.

Miller J.D., Young J.V., Sampson D.R. 1985. Deoxynivalenol and Fusarium head blight resistance in spring cereals. Journal of Phytopathology 113 (4): 359-367.

Oliver R.E., Cai X., Friesen T.L., Halley S., Stack R.W., Xu S.S. 2008. Evaluation of Fusarium head blight resistance in tetraploid wheat (Triticum turgidum L.). Crop Science 48 (1): 213-222. 
Parry D.W., Jenkinson P., Mc Leod L. 1995. Fusarium ear blight (scab) in small grain cereals- a review. Plant Pathology 44 (2): 207-238

Penet L., Guyader S., Pétro D., Salles M., Bussière F. 2014. Direct splash dispersal prevails over indirect and subsequent spread during rains in Colletotrichum gloeosporioides infecting yams. PLOS ONE 9 (12): e115757. DOI 10.1371/journal pone 0115757

Rohácik T., Hudec K. 2005. Influence of agro-environmental factors on Fusarium infestation and population structure in wheat kernels. Annals of Agricultural and Environmental Medicine 12 (1): 39-45.

Rudd J.C., Horsley R.D., McKendry A.L., Elias E.M. 2001. Host plant resistance genes for Fusarium head blight: sources, mechanisms, and utility in conventional breeding systems. Crop Science 41 (3): 620-627.

Sadowski Cz., Lenc L., Wyczling D. 2011. Effect of the forecrop and fungicidal protection on the occurrence of fusarium head blight (Fusarium spp.). Journal of Plant Protection Research 51 (1): 44-49.

Sache I. 2000. Short-distance dispersal of wheat rust spores by wind and rain. Agronomie 20 (7): 757-768.

Shin S., Kim K-H., Kang C-S., Cho K-M., Park C.S., Okagaki R., Park J-C. 2014. A simple method for the assessment of Fusarium head blight resistance in Korean wheat seedlings Inoculated with Fusarium graminearum. Plant Pathology Journal 30 (1): 25-32.

Schroeder H.W., Christensen J.J. 1963. Factors affecting resistance of wheat to scab caused by Gibberella zeae. Phytopathology 53: 831-838.

Siuda R., Grabowski A., Lenc L., Ralcewicz M., Spychaj-Fabisiak E. 2010. Influence of the degree of fusariosis on technological traits of wheat grain. International Journal of Food Science and Technology 45 (12): 2596-2604.

Sneller C., Guttieri M., Paul P., Costa J., Jackwood R. 2012. Variation for resistance to kernel infection and toxin accumulation in winter wheat infected with Fusarium graminearum. Phytopathology 102 (3): 306-314.

Snijders C.H.A., Krechting C.F. 1992. Inhibition of deoxynivalenol translocation and fungal colonization in Fusarium head blight resistant wheat. Canadian Journal of Botany 70 (8): 1570-1576.

Snijders C.H.A., Perkowski J. 1990. Effects of head blight caused by Fusarium culmorum on toxin content and weight of wheat kernels. Phytopathology 80 (6): 566-570.

Steiner B., Lemmens M., Griesser M., Scholz U., Schondelmaier J., Buerstmayr H. 2004. Molecular mapping of resistance to Fusarium head blight in the spring wheat cultivar Frontana. Theoretical and Applied Genetics 109 (1): 215-224.

Steinglein S.A. 2009. Fusarium poae: a pathogen that needs more attention. Journal of Plant Pathology 91 (1): 25-36.

Steyn P.S. 1995. Mycotoxins, general view, chemistry and structure. Toxicology Letters 82-83: 843-851.

Stępień L., Chełkowski J. 2010. Fusarium head blight of wheat: pathogenic species and their mycotoxins. World Mycotoxin Journal 3 (2): 107-119.
Thrane U., Adler A., Clasen P., Galvano F., Langseth W., Lew H., Logrieco A., Nielsen K.F., Ritieni A. 2004. Diversity in metabolite production by Fusarium langsethiae, Fusarium poae, and Fusarium sporotrichioides. International Journal of Food Microbiology 95 (3): 257-266.

Wakuliński W., Chełkowski J. 1993. Fusarium species causing scab of wheat, rye and triticale in Poland. Hodowla Roślin, Aklimatyzacja i Nasiennictwo (Special Edition) 37 (4): 137-142.

Wakuliński W., Solfrizzo M., Perkowski J. 1991. Susceptibility of selected winter wheat cultivars produced in Poland to Fusarium head blight. Mycotoxins Research 7 (1): 91-96.

Wegulo S.N., Bockus W.W., Nopsa J.H, De Wolf E.D., Eskridge K.M., Peiris K.H.S., Dowell S.E. 2011. Effects of integrating cultivar resistance and fungicide application on Fusarium head blight and deoxynivalenol in winter wheat. Plant Disease 95 (5): 554-560.

Wilcoxson R.D., Kommedahl T., Ozmon E.A., Windels C.E. 1988. Occurrence of Fusarium species in scabby wheat from Minnesota and their pathogenicity to wheat. Phytopathology 78 (5): 586-589.

Wiśniewska H., Kowalczyk K. 2005. Resistance of cultivars and breeding lines of spring wheat to Fusarium culmorum and powdery mildew. Journal of Applied Genetics 46 (1): 35-40.

Wiśniewska H., Stępień Ł., Waśkiewicz A., Beszterda M., Góral T., Belter J. 2014. Toxigenic Fusarium species infecting wheat heads in Poland. Central European Journal of Biology 9 (2): 163-172.

Wong L.S.L., Tekauz A., Leisle D., Abramson D., McKenzie R.I.H. 1992. Prevalence, distribution, and importance of Fusarium head blight in wheat in Manitoba. Canadian Journal of Plant Pathology 14 (3): 233-238.

Xu X.M., Monger W., Ritieni A., Nicholson P. 2007. Effect of temperature and duration of wetness during initial infection periods on disease development, fungal biomass and mycotoxin concentrations on wheat inoculated with single, or combinations of, Fusarium species. Plant Pathology 56 (6): 943-956.

Xu X.M., Nicholson P., Thomsett M.A., Simpson D., Cooke B.M., Doohan F.M., Brennan J., Monaghan S., Moretti A., Mule G., Hornok L., Beki E., Tatnell J., Ritieni A., Edwards S.G. 2008. Relationship between the fungal complex causing Fusarium head blight of wheat and environmental conditions. Phytopathology 98 (1): 69-78.

Yang X., Wilson L.L., Madden L.V., Ellis M.A. 1990. Rain splash dispersal of Colletotrichum acutatum from infected strawberry fruit. Phytopathology 80 (6): 590-595.

Yu J.B., Bai, G.H., Cai S.B., Ban T. 2006. Marker-assisted characterization of Asian wheat lines for resistance to Fusarium head blight. Theoretical and Applied Genetics 113 (2): 308-320.

Zadoks J.C., Chang T.T., Konzak C.F. 1974. A decimal code for the growth stages of cereals. Weed Research 14 (6): 415-421.

Zhang J.X., Jin Y., Rudd J.C., Bockelman H.E. 2008. New Fusarium head blight resistant spring wheat germplasm identified in the USDA National Small Grains Collection. Crop Science 48 (1): 223-235. 